\title{
A Novel 9/7 Wavelet Filter banks For Texture Image Coding
}

\author{
Songjun Zhang ${ }^{1}$ \\ School of Mathematics and Statistics \\ Xi'an Jiaotong University \\ Xi'an 710049, China \\ Zhengxing Cheng ${ }^{3}$ \\ School of Mathematics and Statistics \\ Xi'an Jiaotong University \\ Xi'an 710049, China
}

\author{
Guoan Yang ${ }^{2}$ \\ School of electronics and informations \\ Xi'an Jiaotong University \\ Xi'an 710049, China
}

\author{
Huub van de Wetering ${ }^{4}$ \\ The Department of Mathematics and Computer Science \\ Technische Universiteit Eindhoven \\ Eindhoven, Holland
}

\author{
Xiaofeng Fang ${ }^{5}$ \\ School of Mathematics and Statistics \\ Xi'an Jiaotong University \\ Xi'an 710049, China
}

\begin{abstract}
This paper proposes a novel 9/7 wavelet filter bank for texture image coding applications based on lifting a 5/3 filter to a 7/5 filter, and then to a 9/7 filter. Moreover, a one-dimensional optimization problem for the above 9/7 filter family is carried out according to the perfect reconstruction (PR) condition of wavelet transforms and wavelet properties. Finally, the optimal control parameter of the 9/7 filter family for image coding applications is determined by statistical analysis of compressibility tests applied on all the images in the Brodatz standard texture image database. Thus, a new 9/7 filter with only rational coefficients is determined. Compared to the design method of Cohen, Daubechies, and Feauveau, the design approach proposed in this paper is simpler and easier to implement. The experimental results show that the overall coding performances of the new 9/7 filter are superior to those of the CDF 9/7 filter banks in the JPEG2000 standard, with a maximum increase of $0.185315 \mathrm{~dB}$ at compression ratio 32:1. Therefore, this new 9/7 filter bank can be applied in image coding for texture images as the transform coding kernel.
\end{abstract}

Keywords-9/7 wavelet filter banks; image coding; lifting scheme; texture image; Brodatz database.

\section{INTRODUCTION}

Wavelets can effectively be used in several domains including image segmentation, image enhancement, feature extraction, image retrieval, and image coding [1-3]. Although wavelets play an important role in the field of image coding, designing a wavelet kernel for a specific type of image coding, for instance texture images, is still problematic. The CDF 9/7 filter banks of the biorthogonal 9/7 wavelet proposed by Cohen, Daubechies, and Feauveau, is adopted by the JPEG2000 standard as a core algorithm. Although CDF 9/7 has had great impact and has a wide range of applications, its design method is too complicated and its VLSI hardware implementation is too complex. Therefore, this paper proposes new 9/7 filter banks based on Sweldens' lifting scheme [4-6]. Starting from the relatively simple $5 / 3$ filter, this paper presents lifting to a $7 / 5$ filter and subsequently to a 9/7 filter. Then, a one-dimensional parametric $9 / 7$ filter family is derived, as well as providing the dynamic range of control parameters according to Daubechies regularity criterion. Finally, the $9 / 7$ filter family designed in this paper is applied in an image coding application to Brodatz standard texture image database, where a new 9/7 filter bank with the optimal control parameter is determined by maximizing the PSNR (Peak Signal to Noise Ratio).

For the optimal design of biorthogonal wavelets, Cheng, constructed, based on the lifting algorithm, the compact support of biorthogonal wavelet filters and proposed a parametric expression for $9 / 7$ wavelets [7]. In the meantime, Yang designed $9 / 7$ and $7 / 5$ wavelets on the basis of the lifting algorithm [8-9]. The lifting algorithm, presented in [7] and [9] adopts the Euclidean algorithm without providing the lifting operator that can directly improve to a 9/7 wavelet. Phoong and Vaidyanathan proposed the biorthogonal wavelet design method [10]. Antonini and Daubechies designed a wavelet base function for image compression through utilizing the visual features both in the space and frequency domain [11]. Wei and Burrus designed a novel compact support biorthogonal Coifman wavelet in the time domain [12]. The filter design methods mentioned in the above literature are based on traditional Fourier transform and do not use the lifting algorithm. To design a biorthogonal wavelet filter with vanishing moments of arbitrary multiplicity Liu proposed a method that solves trigonometric polynomial equations with two variables on the basis of Diophantine equations [13]. On the basis of filter optimization and median operation, Quan and Ho proposed an efficient lifting scheme to construct biorthogonal wavelet [14] with better compression 
performance than the JPEG2000 standard CDF 9/7 wavelet; however, no optimal filter is provided in the literature for certain type of images. At present, there is relative little literature on texture image coding, yet the study of texture image coding is an important branch of image research, where Brodatz standard texture image database is one of the representative research subjects. The wavelet filter designed in this paper achieves better application performance in the coding of texture images.

This paper is organized as follows: Section 2 provides the basic theories of lifting scheme. Section 3 proposes a 9/7 wavelet filter design approach based on lifting scheme and the Euclidean algorithm and results in a one-dimensional parametric $9 / 7$ wavelet filter. The range of the control variable of the one-dimensional parametric 9/7 wavelet filter designed in section 3 is determined in section 4 . In section 5 this $9 / 7$ wavelet filter is used for the coding of texture images in the Bordatz standard texture image database, an optimal parameter based on the PSNR criterion is determined, and, finally, experimental results for the optimized 9/7 wavelet filter bank are presented and analyzed. Section 5 states the conclusion of this paper and provides implications for future research.

\section{WAVELET LIFTING SCHEME}

This paper focuses on biorthogonal wavelet filters. Let $\{h(z), g(z), \tilde{h}(z), \tilde{g}(z)\}$ be a compactly supported filter bank for such a wavelet. For filters $h(z)$ and $g(z)$, their polyphase representations are:

$$
\begin{aligned}
& h(z)=h_{e}\left(z^{2}\right)+z^{-1} h_{o}\left(z^{2}\right) \\
& g(z)=g_{e}\left(z^{2}\right)+z^{-1} g_{o}\left(z^{2}\right)
\end{aligned}
$$

where

$$
\begin{aligned}
& h_{e}(z)=\sum_{k} h_{2 k} z^{-k}, h_{o}(z)=\sum_{k} h_{2 k+1} z^{-k}, \\
& g_{e}(z)=\sum_{k} g_{2 k} z^{-k}, g_{o}(z)=\sum_{k} g_{2 k+1} z^{-k} .
\end{aligned}
$$

And their polyphase matrix is:

$$
P(z)=\left[\begin{array}{ll}
h_{e}(z) & g_{e}(z) \\
h_{o}(z) & g_{o}(z)
\end{array}\right]
$$

Similarly, we can define a dual polyphase matrix $\tilde{P}(z)$ :

$$
\tilde{P}(z)=\left[\begin{array}{ll}
\tilde{h}_{e}(z) & \tilde{g}_{e}(z) \\
\tilde{h}_{o}(z) & \tilde{g}_{o}(z)
\end{array}\right]
$$

Definition 2.1 If the determinant of the corresponding polyphase matrix $P(z)$ of filter pair $(h, g)$ is 1 , then we say filter pair $(h, g)$ are complementary.
Theorem 2.2 (Lifting) Suppose filter pair $(h, g)$ are complementary, any of the following form of limited filter $g^{\text {new }}$ and $h$ are complementary:

$$
g^{\text {new }}(z)=g(z)+h(z) s\left(z^{2}\right),
$$

where $s(z)$ is a polynomial of Laurent.

Theorem 2.3 (Dual lifting) Suppose filter pair $(h, g)$ are complementary, any of the following form of limited filter $h^{\text {new }}$ and $g$ are complementary:

$$
h^{\text {new }}(z)=h(z)+g(z) t\left(z^{2}\right) \text {, }
$$

where $t(z)$ is a polynomial of Laurent.

For all the polyphase matrices of filter bank $h(z), g(z)$, $\tilde{h}(z), \tilde{g}(z)$, there are:

$$
\begin{aligned}
& P^{n e w}(z)=P(z)\left[\begin{array}{cc}
1 & s(z) \\
0 & 1
\end{array}\right] \\
& \tilde{P}^{\text {new }}(z)=\tilde{P}(z)\left[\begin{array}{cc}
1 & 0 \\
-s\left(z^{-1}\right) & 1
\end{array}\right]
\end{aligned}
$$

where $P^{\text {new }}$ and $\tilde{P}^{\text {new }}$ are the polyphase matrix and the dual polyphase matrix after lifting, respectively.

III 9/7 WAVELET Filter Design Based ON The LIFTING SCHEME

\section{A. Wavelet Lifting from $5 / 3$ to $7 / 5$ filter}

If the filters of a 5/3 wavelet are given by:

$$
\begin{aligned}
& h(z)=h_{2} z^{-2}+h_{1} z^{-1}+h_{0}+h_{1} z+h_{2} z^{2}, \\
& g(z)=g_{1} z^{-1}+g_{0}+g_{1} z,
\end{aligned}
$$

their polyphase representations are, as follows:

$$
\begin{aligned}
& h_{e}(z)=h_{2} z^{-1}+h_{0}+h_{2} z, \\
& h_{o}(z)=h_{1}+h_{1} z, \\
& g_{e}(z)=g_{0}, \\
& g_{o}(z)=g_{1}+g_{1} z .
\end{aligned}
$$

Applying the Euclidean algorithm to $\left\{\begin{array}{l}a_{0}(z)=h_{e}(z) \\ b_{0}(z)=h_{o}(z)\end{array}\right.$ may give in two steps the following quotients $q_{i}$ and remainders $r_{i}(i=1,2)$ : 


$$
\left\{\begin{array}{l}
q_{1}(z)=\frac{h_{2}}{h_{1}}\left(1+z^{-1}\right) \\
q_{2}(z)=\frac{h_{1}}{h_{0}-2 h_{2}}(1+z) \\
r_{1}(z)=h_{0}-2 h_{2} \\
r_{2}(z)=0
\end{array}\right.
$$

Given these quotients the polyphase matrix $P(z)$ can be factorized:

$$
P(z)=\left[\begin{array}{ll}
h_{e} & g_{e} \\
h_{o} & g_{o}
\end{array}\right]=\prod_{i=1}^{2}\left[\begin{array}{cc}
q_{i}(z) & 1 \\
1 & 0
\end{array}\right]\left[\begin{array}{cc}
K & 0 \\
0 & 1 / K
\end{array}\right]
$$

where $K$ is a constant scale factor.

For $\alpha=\frac{h_{2}}{h_{1}}, \beta=\frac{h_{1}}{h_{0}-2 h_{2}}$, equation (6) gives the following polyphase matrix:

$$
\begin{aligned}
& P(z)=\left[\begin{array}{ll}
h_{e}(z) & g_{e}(z) \\
h_{o}(z) & g_{o}(z)
\end{array}\right] \\
& =\left[\begin{array}{cc}
K\left(\alpha \beta z+2 \alpha \beta+1+\alpha \beta z^{-1}\right) & \alpha\left(1+z^{-1}\right) / K \\
K \beta(1+z) & 1 / K
\end{array}\right]
\end{aligned}
$$

Let lifting operator $s(z)$ be:

$$
s(z)=K^{-2} \gamma\left(1+z^{-1}\right),
$$

where $\gamma$ is a free parameter. The polyphose matrix $P^{\text {new }}(z)$ for the $7 / 5$ filter can now be given by:

$$
\begin{aligned}
& P^{\text {new }}(z)=P(z)\left[\begin{array}{cc}
1 & s(z) \\
0 & 1
\end{array}\right] \\
& =\left[\begin{array}{ll}
h_{e}(z) & h_{e}(z) s(z)+g_{e}(z) \\
h_{o}(z) & h_{o}(z) s(z)+g_{o}(z)
\end{array}\right] .
\end{aligned}
$$

After lifting we obtain the new $7 / 5$ filter coefficients:

$$
\left\{\begin{array}{l}
h_{0}=(1+2 \beta \gamma) / K \\
h_{1}=(3 \alpha \beta \gamma+\gamma+\alpha) / K \\
h_{2}=\beta \gamma / K \\
h_{3}=\alpha \beta \gamma / K \\
g_{0}=(2 \alpha \beta+1) K \\
g_{1}=\beta K \\
g_{2}=\alpha \beta K
\end{array}\right.
$$

Wavelet Lifting from 7/5 to 9/7 filter
If the filter of the $7 / 5$ wavelet filter is

$$
\begin{aligned}
& h(z)=h_{3} z^{-3}+h_{2} z^{-2}+h_{1} z^{-1}+h_{0}+h_{1} z+h_{2} z^{2}+h_{3} z^{3}, \\
& g(z)=g_{2} z^{-2}+g_{1} z^{-1}+g_{0}+g_{1} z+g_{2} z^{2},
\end{aligned}
$$

their polyphase representations are given by:

$$
\begin{aligned}
& h_{e}=h_{0}+h_{2}\left(z+z^{-1}\right), \\
& h_{o}=h_{1}(z+1)+h_{3}\left(z^{2}+z^{-1}\right), \\
& g_{e}(z)=g_{0}+g_{2}\left(z+z^{-1}\right), \\
& g_{o}(z)=g_{1}+g_{1} z .
\end{aligned}
$$

Again applying the Euclidean algorithm to $\left\{\begin{array}{l}a_{0}(z)=h_{e}(z) \\ b_{0}(z)=h_{0}(z)\end{array}\right.$ $b_{0}(z)=h_{o}(z)$ may give in four steps the following quotients $q_{i}$ and remainders $r_{i}(i=1,2,3,4)$ :

$$
\left\{\begin{array}{l}
q_{1}(z)=0, \quad r_{1}(z)=h_{0}+h_{2}\left(z+z^{-1}\right) \\
q_{2}(z)=\frac{h_{3}}{h_{2}}(1+z), \quad r_{2}(z)=\left(h_{1}-h_{3}-\frac{h_{0} h_{3}}{h_{2}}\right)(1+z) \\
q_{3}(z)=\frac{h_{2}}{h_{1}-h_{3}-\frac{h_{0} h_{3}}{h_{2}}}\left(1+z^{-1}\right), \quad r_{3}(z)=h_{0}-2 h_{2} \\
q_{4}(z)=\frac{h_{1}-h_{3}-\frac{h_{0} h_{3}}{h_{2}}}{h_{0}-2 h_{2}}(1+z), \quad r_{4}(z)=0
\end{array}\right.
$$

The corresponding polyphase matrix factorization is:

$$
\begin{aligned}
& P(z)=\left[\begin{array}{ll}
h_{e}(z) & g_{e}(z) \\
h_{o}(z) & g_{o}(z)
\end{array}\right] \\
& =\prod_{i=1}^{4}\left[\begin{array}{cc}
q_{i}(z) & 1 \\
1 & 0
\end{array}\right]\left[\begin{array}{cc}
K & 0 \\
0 & 1 / K
\end{array}\right]
\end{aligned}
$$

For $\alpha=\frac{h_{3}}{h_{2}}, \beta=\frac{h_{2}}{h_{1}-h_{3}-\frac{h_{0} / h_{3}}{h_{2}}}, \gamma=\frac{h_{1}-h_{3}-\frac{h_{0} / h_{2}}{h_{2}}}{h_{0}-2 h_{2}}$, according to (10) we get the following matrix for $P(z)$ :

$$
\begin{aligned}
& P(z)= \\
& {\left[\begin{array}{cc}
K\left(\beta \gamma z^{-1}+2 \beta \gamma+1+\beta \gamma z\right) & \beta\left(1+z^{-1}\right) / K \\
K\left(\alpha \beta \gamma z^{-1}+3 \alpha \beta \gamma+\gamma+\alpha\right. & \left(\alpha \beta z^{-1}+2 \alpha \beta+1+\alpha \beta z\right) / K \\
\left.+(3 \alpha \beta \gamma+\gamma+\alpha) z+\alpha \beta \gamma z^{2}\right) &
\end{array}\right]}
\end{aligned}
$$

The filters $h(z)$ and $g(z)$ now follow from the polyphase form: 


$$
\begin{aligned}
h(z)= & h_{e}\left(z^{2}\right)+z^{-1} h_{o}\left(z^{2}\right) \\
= & K\left(\beta \gamma z^{-2}+2 \beta \gamma+1+\beta \gamma z^{2}\right) \\
& +z^{-1} K\left(\alpha \beta \gamma z^{-2}+3 \alpha \beta \gamma+\gamma+\alpha\right. \\
& \left.+(3 \alpha \beta \gamma+\gamma+\alpha) z^{2}+\alpha \beta \gamma z^{4}\right) \\
= & K\left(\alpha \beta \gamma z^{-3}+\beta \gamma z^{-2}+(3 \alpha \beta \gamma+\gamma+\alpha) z^{-1}\right. \\
& \left.+2 \beta \gamma+1+(3 \alpha \beta \gamma+\gamma+\alpha) z+\beta \gamma z^{2}+\alpha \beta \gamma z^{3}\right) \\
g(z)= & g_{e}\left(z^{2}\right)+z^{-1} g_{o}\left(z^{2}\right) \\
= & \beta\left(1+z^{-2}\right) / K \\
& +z^{-1}\left(\alpha \beta z^{-2}+2 \alpha \beta+1+\alpha \beta z^{2}\right) / K \\
= & \left(\alpha \beta z^{-3}+\beta z^{-2}+(2 \alpha \beta+1) z^{-1}+\beta+\alpha \beta z\right) / K
\end{aligned}
$$

With the lifting operator with free parameter $\eta$ given by the following equation

$$
s(z)=K^{-2} \eta\left(1+z^{-1}\right),
$$

the new polyphase matrix is obtained as follows:

$$
\begin{aligned}
& P^{n e w}(z)=P(z)\left[\begin{array}{cc}
1 & s(z) \\
0 & 1
\end{array}\right] \\
& =\left[\begin{array}{ll}
h_{e}(z) & h_{e}(z) s(z)+g_{e}(z) \\
h_{o}(z) & h_{o}(z) s(z)+g_{o}(z)
\end{array}\right]
\end{aligned}
$$

So, the resulting $9 / 7$ coefficients after lifting are:

$$
\left\{\begin{array}{l}
h_{0}=(6 \alpha \beta \gamma \eta+2 \eta \gamma+2 \eta \alpha+2 \alpha \beta+1) / K \\
h_{1}=(3 \beta \gamma \eta+\eta+\beta) / K \\
h_{2}=(4 \alpha \beta \gamma \eta+\gamma \eta+\alpha \eta+\alpha \beta) / K \\
h_{3}=\beta \gamma \eta / K \\
h_{4}=\alpha \beta \gamma \eta / K \\
g_{0}=(1+2 \beta \gamma) K \\
g_{1}=(3 \alpha \beta \gamma+\gamma+\alpha) K \\
g_{2}=\beta \gamma K \\
g_{3}=\alpha \beta \gamma K
\end{array}\right.
$$

According to the perfect reconstruction condition of $9 / 7$ wavelet transform, wavelet properties, and normalizing condition, the above coefficients can be expressed in the form of a one-dimensional function:

$$
\left\{\begin{array}{l}
h_{0}=-\left(8 t^{3}-18 t^{2}+7 t-20\right) / 16 t \\
h_{1}=\left(4 t^{3}-11 t^{2}+15 t-4\right) / 8 t \\
h_{2}=(t-2) / 4 t \\
h_{3}=\left(4 t^{2}-7 t+4\right)(t-1) / 8 t \\
h_{4}=\left(4 t^{2}-7 t+4\right)(2 t-1) / 32 t \\
g_{0}=(t+1) / 4 \\
g_{1}=(2 t+7) / 32 \\
g_{2}=-(t-1) / 8 \\
g_{3}=-(2 t-1) / 32
\end{array}\right.
$$

Based on equation (14), the range of $t$ can be determined as $t \in[0.78,1.85]$. If $t$ is a known number, the filter coefficients can be easily be determined using equation (14), resulting in a newly designed 9/7 wavelet filter bank.

\section{THE REgULARITY OF 9/7 WAVELET}

The biorthogonal wavelet filter bank $\{h(z), g(z), \tilde{h}(z), \tilde{g}(z)\}$ must satisfy the regularity condition for image coding. Suppose $L_{1}$ and $L_{2}$ are the vanishing moments of lowpass filter on analysis and synthesis sides respectively, the dynamic range of the control variable $t$ in equation (14) is determined by adopting Daubechies' theorem. We have the following equations:

$$
\left\{\begin{array}{l}
h(z)=\left[\frac{\left(1+e^{-i \xi}\right)}{2}\right]^{L_{1}} F(\xi) \\
g(z)=\left[\frac{\left(1+e^{-i \xi}\right)}{2}\right]^{L_{2}} Q(\xi)
\end{array}\right.
$$

where $z=e^{i \xi}, F(\xi)$ and $Q(\xi)$ are both trigonometric polynomial related with control variable. Concurrently, we get the following equations:

$$
\left\{\begin{array}{l}
B_{k}^{1}=\max _{\xi}\left|F(\xi) F(2 \xi) \cdots F\left(2^{k_{1}-1} \xi\right)\right|^{1 / k_{1}}<2^{L_{1}-1 / 2} \\
B_{k}^{2}=\max _{\xi}\left|Q(\xi) Q(2 \xi) \cdots Q\left(2^{k_{2}-1} \xi\right)\right|^{1 / k_{2}}<2^{L_{2}-1 / 2},
\end{array}\right.
$$

where $k_{1}$ and $k_{2}$ are both Integer. The equation (16) gives a limiting condition of biorthogonal wavelet filter bank for image coding application, which leads to the determination of the dynamic range of the control variable $t$ of the new 9/7 wavelet filter family designed in this paper, thus being capable of designing an optimal new 9/7 wavelet filter for image coding. 
As to the new $9 / 7$ wavelet filter designed in this paper, the values of its vanishing moments $L_{1}$ and $L_{2}$ are 2 and 4 . If $k_{1}=k_{2}=40$, according to the equation (16), the range of $t$ can be determined as $t \in[0.78,1.85]$. If $t$ is determined, the filter coefficients can be obtained according to equation (14), thus the new 9/7 wavelet filter can also be determined.

\section{$\mathrm{V}$ EXPERIMENT AND ANALYSIS}

Taking the above one-dimensional parameterized 9/7 wavelet filter family as the coding kernel, adopting EBCOT (Embedded Block Coding with Optimized Truncation) coding [15], and applying this system to the image coding of Brodatz standard texture image database, it is found, by analysis of the experiment statistics in Fig. 1 and Fig. 2, that the control variable at the optimal PSNR is $t=1.2050$. Therefore, our new $9 / 7$ wavelet filter banks for texture image coding applications is known as given in Table1.

The 111 images in Brodatz standard texture image database are all tested based on the above image coding system. The results show that comparing the coding system of JPEG2000 with the coding system proposed in this paper, when compression rate is $4: 1$, the average PSNR value of the 111 texture image in Brodatz is only $0.0077 \mathrm{~dB}$ lower than that of JPEG2000, with the PSNR of 49 images higher than that of JPEG200 at the average height of $0.0373 \mathrm{~dB}$, while the PSNR of 62 images are lower than that of JPEG2000 at the average amount of $0.0433 \mathrm{~dB}$.

When the compression rate are 8:1, 16:1, 32:1, 64:1, and 128:1, respectively, the results show in Table 2.

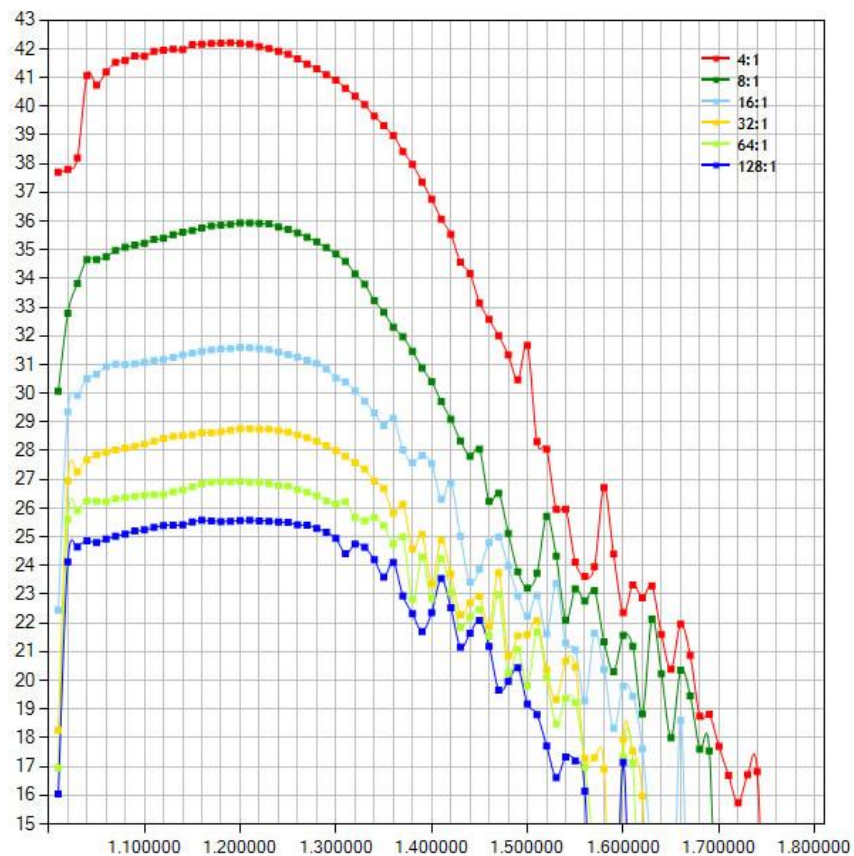

Figure 1. The PSNR value of test image D29 at the control variable's varying range of 1.000000-1.800000 under each compression ratio in the Brodatz standard texture image database
TABLE I. THE COEFFICIENTS OF NEW 9/7 WAVELET FILTER BANK ( $t=1.2050$ )

\begin{tabular}{|l|l|l|l|c|}
\hline \multirow{2}{*}{$\mathrm{k}$} & \multicolumn{2}{|c|}{ Analysis filter coefficients } & \multicolumn{2}{c|}{ Synthesis filter coefficients } \\
\cline { 2 - 5 } & $\begin{array}{c}\text { Low pass } \\
h_{k}\end{array}$ & High pass $\tilde{h}_{k}$ & $\begin{array}{c}\text { Low pass } \\
g_{k}\end{array}$ & $\begin{array}{c}\text { Hign pass } \\
\tilde{g}_{k}\end{array}$ \\
\hline 0 & 0.5513 & 1.2295 & 1.2295 & 0.5513 \\
\hline \pm 1 & 0.2941 & -0.5292 & 0.5292 & -0.2941 \\
\hline \pm 2 & -0.0256 & -0.1649 & -0.1649 & -0.0256 \\
\hline \pm 3 & -0.0441 & -0.0292 & 0.0292 & 0.0441 \\
\hline \pm 4 & & 0.0502 & 0.0502 & \\
\hline
\end{tabular}

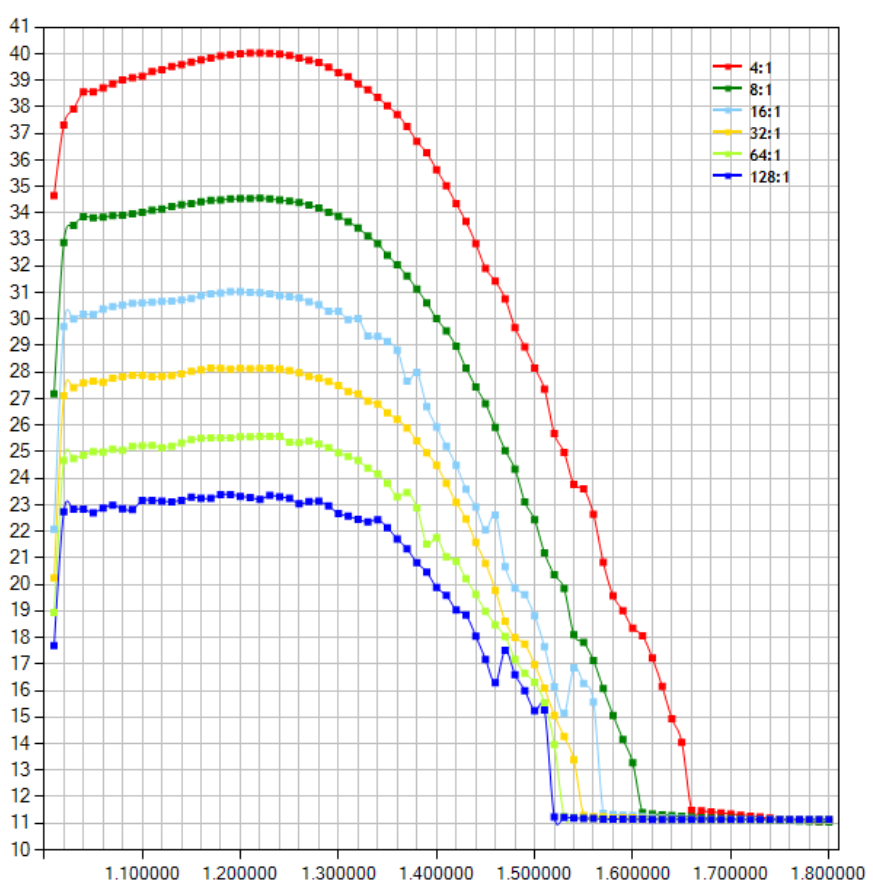

Figure 2. The PSNR value of image D110 at the control variable's varying range of 1.000000-1.800000 under each compression ratio in the Brodatz standard texture image database

The objective comparison of experimental statistic of maximum PSNR under different compression ratio for test images in the Brodatz standard texture image database are shown in Table 3.

The subjective comparison of compression performances between our new 9/7 filter and CDF 9/7 for test images D29, D43, D84 and D103 in the Brodatz standard texture image database under compression ratio 32:1 are shown in Figure 3, Figure 4 and Figure 5.

The images in Fig.4 are the reconstructed images of the above four images through the compression experiments by JPEG2000 standard CDF 9/7 filter at the compression ratio of $32: 1$.

The images in Fig.5 are the reconstructed images of same four images from the Brodatz standard texture image database, but through the compression experiments by the new $9 / 7$ filter designed in this paper at the compression ratio of 32:1. 
From comparison of Fig.4 and Fig.5, the resulting subjective visual quality of reconstructed images using the new $9 / 7$ filter is concluded to be as good as the quality resulting from using the CDF 9/7 filter.

TABLE II. COMPARISON OF THE COMPRESSION PERFORMANCES BETWEEN NEW 9/7 AND CDF 9/7 FILTER (PSNR/DB)

\begin{tabular}{|l|c|c|c|c|c|c|c|}
\hline \multicolumn{1}{|c|}{$\begin{array}{c}\text { Image } \\
\text { Database }\end{array}$} & $\begin{array}{c}\text { Compressi } \\
\text { on Ratio }\end{array}$ & $\begin{array}{c}\text { Total } \\
\text { amount of } \\
\text { images }\end{array}$ & $\begin{array}{c}\text { Mean } \\
\text { differences }\end{array}$ & $\begin{array}{c}\text { Image } \\
\text { number }\end{array}$ & $\begin{array}{c}\text { Mean } \\
\text { differences }\end{array}$ & $\begin{array}{c}\text { Image } \\
\text { number }\end{array}$ & $\begin{array}{c}\text { Mean } \\
\text { differences }\end{array}$ \\
\hline Brodatz & $4: 1$ & 111 & -0.0077 & 49 & +0.0373 & 62 & -0.0433 \\
\hline Brodatz & $8: 1$ & 111 & +0.0014 & 52 & +0.0262 & 59 & -0.0205 \\
\hline Brodatz & $16: 1$ & 111 & +0.0028 & 64 & +0.0231 & 47 & -0.0248 \\
\hline Brodatz & $32: 1$ & 111 & +0.0207 & 75 & +0.0483 & 36 & -0.0369 \\
\hline Brodatz & $64: 1$ & 111 & +0.0330 & 82 & +0.0593 & 29 & -0.0415 \\
\hline Brodatz & $128: 1$ & 111 & +0.0482 & 70 & +0.1072 & 41 & -0.0525 \\
\hline
\end{tabular}

TABLE III. EXPERIMENT STATISTICS OF MAXIMUM PSNR FOR DIFFERENT COMPRESSION RATIOS

\begin{tabular}{|c|c|c|c|c|}
\hline Compression Ratio & Image & CDF9/7 wavelet & New $9 / 7$ wavelet & Difference \\
\hline \multirow{4}{*}{$4: 1$} & D110 & 27.595602 & 27.670151 & +0.074549 \\
\hline & D71 & 32.500939 & 32.574722 & +0.073783 \\
\hline & D91 & 44.501023 & 44.570713 & +0.069690 \\
\hline & D52 & 34.765339 & 34.833791 & +0.068452 \\
\hline \multirow{4}{*}{$8: 1$} & D103 & 22.897491 & 22.982836 & +0.085345 \\
\hline & D5 & 28.339782 & 28.408767 & +0.068985 \\
\hline & D47 & 35.412800 & 35.480066 & +0.067266 \\
\hline & $\mathrm{D} 2$ & 26.703806 & 26.761880 & +0.058074 \\
\hline \multirow{4}{*}{$16: 1$} & D47 & 30.651179 & 30.727201 & +0.076022 \\
\hline & D62 & 31.821213 & 31.886339 & +0.065126 \\
\hline & D98 & 30.184702 & 30.247055 & +0.062353 \\
\hline & D38 & 26.300726 & 26.354982 & +0.054256 \\
\hline \multirow{4}{*}{$32: 1$} & D103 & 15.796872 & 15.982187 & +0.185315 \\
\hline & D84 & 18.952107 & 19.079902 & +0.127795 \\
\hline & $\mathrm{D} 29$ & 21.238714 & 21.347855 & +0.109141 \\
\hline & D43 & 28.080872 & 28.189965 & +0.109093 \\
\hline \multirow{4}{*}{$64: 1$} & D87 & 15.424513 & 15.692062 & +0.267549 \\
\hline & D30 & 28.624222 & 28.829037 & +0.204815 \\
\hline & D102 & 17.269489 & 17.456338 & +0.186849 \\
\hline & D18 & 21.291975 & 21.457116 & +0.165141 \\
\hline \multirow{4}{*}{$128: 1$} & D53 & 13.688657 & 14.018516 & +0.329859 \\
\hline & D65 & 17.517933 & 17.817868 & +0.299935 \\
\hline & D68 & 18.748988 & 19.044899 & +0.295911 \\
\hline & D46 & 23.911123 & 24.167780 & +0.256657 \\
\hline
\end{tabular}



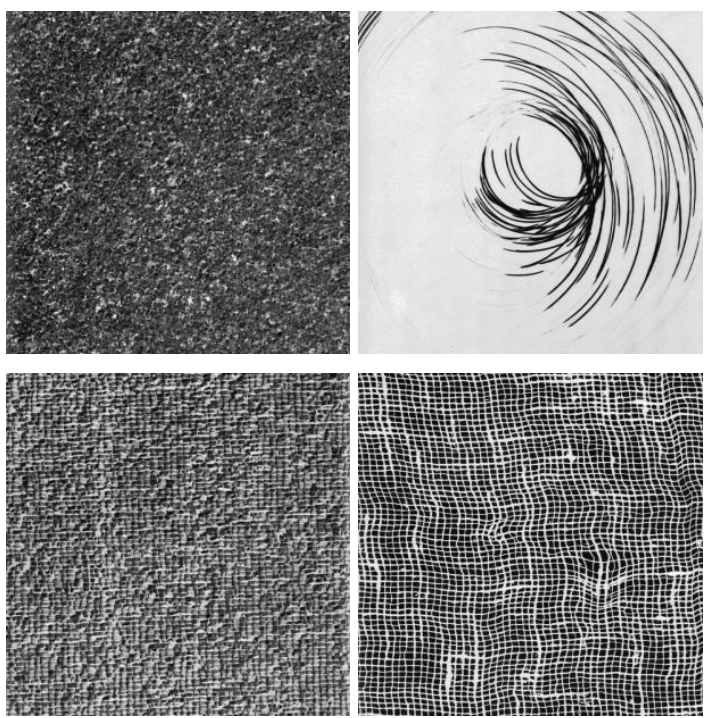

Figure 3. Original four images, Top: D29 and D43, Bottm: D84 and D103
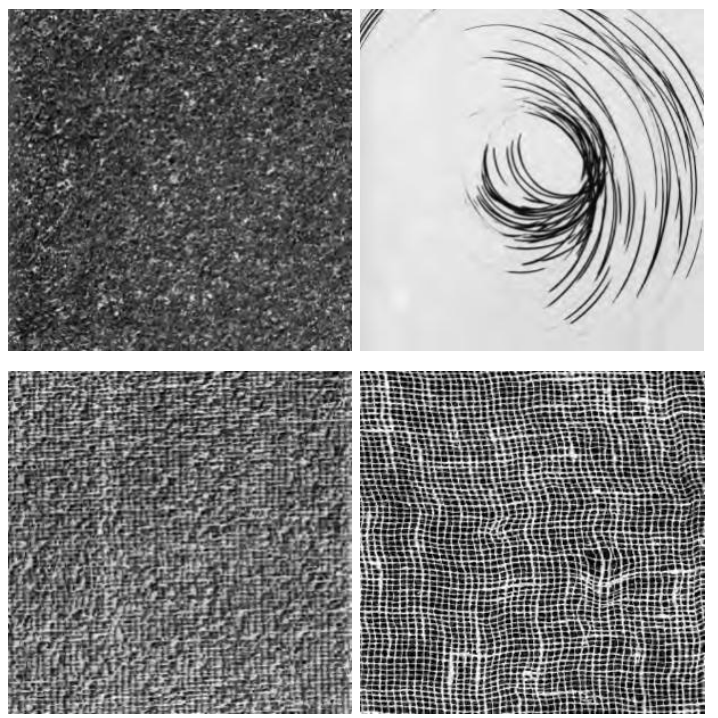

Figure 4. The reconstructed images, Top: D29 and D43, Bottm: D84 and D103, CDF9/7, CR=32:1

\section{CONCLUSION}

Compared with CDF 9/7 wavelet filter, the new 9/7 wavelet filter designed in this paper is much easier to be constructed and more favorable in hardware implementation. The results show that under high compression ratio (low bit rate), the overall coding performance of the new $9 / 7$ wavelet filter is better than that of the JPEG2000 CDF9/7 wavelet filter, therefore, the $9 / 7$ wavelet filter designed in this paper is very effective in image coding for texture image.

\section{ACKNOWLEDGMENT}

This work was supported by the Chinese national natural science foundation under grant 51075317, and project 973 of national key basic research of China under grant 2007CB311005.
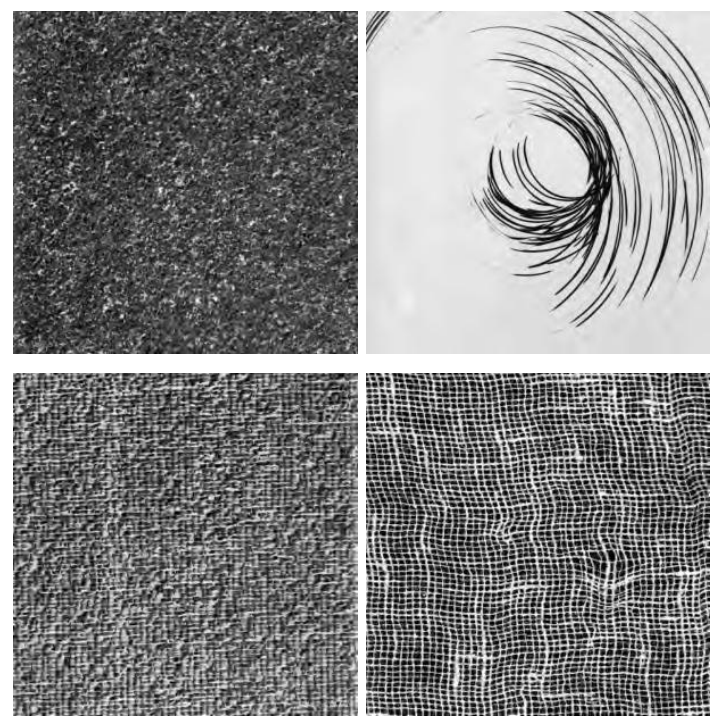

Figure 5. The reconstructed images, Top: D29 and D43, Bottm: D84 and D103, new $9 / 7, \mathrm{CR}=32: 1$

\section{REFERENCES}

[1] M. Vertterli, and J. Kovacevic, Wavelet and subband coding. Prentice Hall, Englewood Cliffs, New Jersey, 1995

[2] M. Vetterli, and C. Herley, "Wavelets and Filter Banks: Theory and Design," IEEE Transactions on Signal Processing, vol.40, 1992, pp.2207-2232.

[3] A. Cohen, I. Daubechies, and J. Feauveau, "Biorthogonal bases of compactly supported wavelets," Communications on Pure and Applied Mathematics, vol.45, 1992, pp.485-560.

[4] W. Sweldens, "The lifting scheme: a construction of second generation wavelets," SIAM Journal on Mathematical Analysis, vol.29, 1997, pp.511-546.

[5] W. Sweldens, "The lifting scheme: a custom-design construction of biorthogonal wavelets," Applied and Computational Harmonic Analysis, vol.3, 1996, pp.186-200.

[6] I. Daubechies, and W. Sweldens, "Factoring Wavelet Transforms into Lifting Steps," The Journal of Fourier Analysis and Applications, vol.4, 1998, pp.247-269.

[7] L. Cheng, D.L. Liang, and Z.H. Zhang, "Popular biorthogonal wavelet filters via a lifting scheme and its application in image compression," IEE Proceedings Vision, Image and Signal Processing, vol.150, 2003, pp.227-232.

[8] Guoan Yang, and Nanning Zheng, "An Optimization Algorithm for Biorthogonal Wavelet Filter Banks Design," International Journal of Wavelets, Multiresolution and Information Processing, vol.6, 2008, pp.51-63.

[9] Guoan Yang, Nanning Zheng, and Shugang Guo, "Optimal Wavelet Filter Design for Remote Sensing Image Compression," Journal of Electronics (China), vol.24, 2007, pp.276-284.

[10] See-May Phoong, C.W. Kim, P.P. Vaidyanathan, and R. Ansari, "A new class of two-channel biorthogonal filter banks and wavelet bases," IEEE Transactions on Signal Processing, vol.43, 1995, pp.649-665.

[11] M. Antonini, M. Barlaud, P. Mathieu, and I. Daubechies, "Image coding using wavelet transform," IEEE Transactions on Image processing, vol.1, 1992, pp.205-220.

[12] Dong Wei, Jun Tian, R.O. Wells, and C.S. Burrus, "A new class of biorthognal wavelet systems for image transform coding," IEEE Transactions on Image processing, vol.7, 1998, pp.1000-1013.

[13] Zaide Liu, Nanning Zheng, Yuehu Liu, and Huub van de Wetering, "Optimization Design of Biorthogonal Wavelets for Embedded Image Coding," IEICE Transactions on Information and Systems, vol.E90-D, 2007, pp.569-578. 
[14] Do Quan, and Yo-Sung Ho, "Efficient Wavelet Lifting Scheme Based on Filter Optimization and Median Operator," IEEE-RIVF International Conference on Computing and Communication Technologies, Da Nang Univ Technol, Da Nang, Vietnam, 2009, pp.284-289.

[15] D. Taubman, "High performance scalable image compression with EBCOT," IEEE Transactions on Image processing, vol.9, 2000, pp.1158-1170.

[16] A. Said, and W.A. Pearlman, "A new fast and efficient image codes based set partitioning in hierarchical trees," IEEE Transactions on Circuits and Systems for Video Technology, vol.6, 1996, pp.243-250.

[17] L. F. Villemoes, "Energy Moments in Time and Frequency for TwoScale Difference Equation Solutions and Wavelets," SIAM Journal on Mathematical Analysis, vol.23, 1992, pp.1519-1543.
[18] A. Cohen, and I. Daubechies, "A Stability Criterion For Biorthoronal Wavelet Bases And Their Related Subband Coding Scheme," DUKE Mathematical Journal, vol.68, 1992, pp.313-335.

[19] M. Unser, and T. Blu, "Mathematical properties of the JPEG2000 wavelet filters. IEEE Transactions on Image processing, 2003, 12(9): 1080-1090.

[20] J. D. Villasenor, B. Belzer, and J. Liao, "Wavelet filter evaluation for image compression," IEEE Transactions on Image Processing, vol.4, 1995, pp.1053-1060.

[21] Yi Shang, Longzhuang Li, and Benjamin Wah, "Optimization design of biorthogonal filter banks for image compression," Elsevier Information Sciences, vol.132, 2001, pp.23-51. 